\title{
INTELIGÊNCIA DE NEGÓCIOS E SUA CONDIÇÃO EPISTEMOLÓGICA NA CIÊNCIA DA INFORMAÇÃO
}

\section{BUSINESS INTELLIGENCE Y SU CONDICIÓN EPISTEMOLÓGICA EN CIENCIAS DE LA INFORMACIÓN}

\author{
Alexandre Lucasa \\ Angel Freddy Godoy Vierab \\ William Barbosa Viannac
}

\begin{abstract}
RESUMO
Introdução: A Inteligência de Negócios ou Business Intelligence (BI) é um termo bastante empregado em diversos universos e apresenta imprecisões sobre a sua definição e suas delimitações epistemológicas. A Ciência da Informação tradicionalmente tem investigado as propriedades e o comportamento informacional, as forças que governam os fluxos de informação, e os significados do processamento da informação, visando à acessibilidade e a usabilidade ótima. Nesse sentido, pergunta-se: A Ciência da Informação possui suporte epistemológico para contribuir com o desenvolvimento de abordagens de Inteligência de Negócios? Objetivo: $O$ objetivo desse estudo é identificar os fundamentos epistemológicos que permitem abordar a Inteligência de Negócios no campo da Ciência da Informação. Metodologia: O estudo, de natureza exploratória, busca ampliar o entendimento do tema por meio de uma revisão conceitual sobre as origens, a epistemologia e abrangência da Ciência da Informação e as interconexões com a Inteligência de Negócios. Resultados: $O$ resultado identifica as potencialidades da Ciência da Informação para com a Inteligência de Negócios por meio de seus paradigmas e modelos de estudo dos conhecimentos. Conclusões: O paradigma cognitivo - seu modelo de estudo, pesquisadores e teorias da informação - é apresentado como o mais especificamente apropriado para o suporte epistemológico de Inteligência de Negócios no campo da Ciência da Informação.
\end{abstract}

Descritores: Inteligência de Negócios. Ciência da Informação. Epistemologia.

\footnotetext{
a Doutorando em Ciência da Informação pela Universidade Federal de Santa Catarina. E-mail: alexlucas.al@gmail.com

b Doutor em Engenharia de Produção pela Universidade Federal de Santa Catarina. Professor da Universidade Federal de Santa Catarina. E-mail: recinfor@gmail.com

c Doutor em Engenharia de Produção pela Universidade Federal de Santa Catarina. Professor da Universidade Federal de Santa Catarina. E-mail: wpwilliam@hotmail.com
} 


\section{INTRODUÇÃO}

A Inteligência de negócios ou Business Intelligence (BI) é um termo bastante empregado no universo da Tecnologia da Informação e Comunicação (TIC), mas ainda existem imprecisões sobre a sua definição e suas delimitações epistemológicas, sendo um assunto tratado de diversas formas nas áreas de administração, computação, estatística, entre outras.

Entretanto há certo consenso que inteligência de negócios não é propriamente um produto ou sistema, mas um termo que combina diversas dimensões como arquiteturas, aplicações e bases de dados que permitam a análise, manipulação e acesso interativo, o tanto quanto possível em tempo real, de informações relevantes para tomada de decisão.

Do ponto de vista mais mercadológico, o termo Business Intelligence, surgiu em 1996, a partir do Gartner Research Group sendo concretizado por meio de diferentes sistemas. O Gartner, Inc. (NYSE: IT) é líder mundial no fornecimento de pesquisas e aconselhamento na área de tecnologia da informação. Fundado em 1979, o Gartner tem sede em Stamford, Connecticut, EUA e, possui 6.800 associados, sendo mais de 1.000 analistas de pesquisa e consultores, e clientes em 90 países (GARTNER, 2015).

Turban, Sharda e Delen (2010) definem IN como um termo "guardachuvas" que combina arquiteturas, ferramentas, bases de dados, ferramentas analíticas, aplicações e metodologias.

Para Santos e Ramos (2006), os sistemas de IN combinam o levantamento de dados operacionais que permitam $\mathrm{O}$ seu posterior armazenamento em repositórios adequados para análise, exploração e apresentação de informação essencial para a tomada de decisão.

Segundo Shollo e Galliers (2015), IN são sistemas integrados e ligados a um armazém de dados e outras aplicações. São projetados para facilitar a análise dos dados armazenados (em tempo real e histórico) para apoio a tomada de decisões gerenciais (DAVENPORT, 2006 apud SHOLLO; GALLIERS, 2015). Sistemas de IN diferem de seus antecessores, pois a provisão de informações é habilitada pela integração dos bancos de dados de 
diferentes domínios do negócio. A ideia é que a disponibilidade e a combinação de dados de diferentes fontes podem fornecer novas percepções que podem levar a uma melhor tomada de decisões. Luhn (1958 apud SHOLLO; GALLIERS, 2015) foi o primeiro a utilizar o termo Business Intelligence em um contexto científico. Ele define IN como "a capacidade de apreender as interrelações dos fatos apresentados, de forma a orientar a ação para a meta desejada". Sistemas de IN consistem de processos, tecnologias e aplicações que se destinam a permitir que as organizações para coletar, armazenar, analisar e transformar dados em informações que são relevantes para a tomada de decisões (WIXOM et al, 2010; DAVENPORT et al, 2012 apud SHOLLO; GALLIERS, 2015).

Tendo em vista a abrangência do termo, esse estudo considera que Inteligência de Negócios: (i) trabalha com a informação relevante da organização ou informação estratégica (ii) apoia os processos de tomada de decisões nas organizações; (iii) coleta, organiza, compartilha e permite o monitoramento de informações de suporte as ações preventivas ou corretivas num negócio empresarial.

Por outro lado, conforme Shera e Cleveland (1977), a ciência da informação tradicionalmente tem investigado as propriedades $e \quad 0$ comportamento informacional, as forças que governam os fluxos de informação, e os significados do processamento da informação, visando à acessibilidade e a usabilidade ótima. Desta forma, a Ciência da Informação está preocupada com o corpo de conhecimentos relacionados à origem, coleção, organização, armazenamento, recuperação, interpretação, transmissão, transformação, e utilização da informação. Isto inclui a pesquisa sobre a representação da informação em ambos os sistemas, tanto naturais quanto artificiais, o uso de códigos para a transmissão eficiente da mensagem, bem como o estudo do processamento e de técnicas aplicadas aos computadores e seus sistemas de programação (SHERA; CLEVELAND, 1977).

Nesse sentido, pergunta-se: A Ciência da Informação possui suporte epistemológico para contribuir com o desenvolvimento de abordagens de IN? 


\section{EPISTEMOLOGIA DA CIÊNCIA DA INFORMAÇÃO}

Estudar a Epistemologia da Ciência da Informação (CInf) é estudar as origens, a estrutura, os métodos e os processos de validação que tornam a CInf uma ciência. E, como tal, é importante entender como funciona o seu sistema de adquirir conhecimento, o seu método científico e como se organiza o seu corpo de conhecimento, estabelecido por meio das pesquisas científicas de sua área.

Um modo para estudar a Epistemologia da CInf acontece por meio da análise de textos de autores/pesquisadores desta Ciência, identificando as abrangências, potencialidades e limitações do conceito, buscando um entendimento e contribuições. Porém, entender de partida o que é Epistemologia e a constituição de um campo cientifico ou ciência, é fundamental. Segundo Greco (2008), a epistemologia ou teoria do conhecimento, é conduzida por duas questões principais: $O$ que é 0 conhecimento? O que podemos conhecer? Dessas duas questões decorre uma terceira: Como conhecemos o que conhecemos? Já para Kornblith (apud GRECO, 2008) a epistemologia se preocupa com três questões: O que é conhecimento? Como o conhecimento é possível? O que devemos fazer para obter conhecimento?

Borges et al (2004) questionam como é possível, para um observador, um cientista, descrever um fenômeno ou evento que ocorre no mundo, sendo ele mesmo, a linguagem, as teorias e o que quer que seja, parte integrante deste mundo? A resposta, embora não seja a única admissível, é que isto só será possível com o reconhecimento e a aceitação de que as explicações científicas têm um caráter tautológico, ou seja, explicando-se de diversas maneiras a mesma teoria, é possível gerar o conhecimento cientificamente aceitável.

Cabe ainda ressaltar que, conforme Pinheiro (2005), o aparecimento de um campo do conhecimento pode ser percebido por alguns eventos legitimadores, como: reuniões, periódicos e sociedades científicas, instituídos e dedicados à nova área. Harmon (1971 apud PINHEIRO, 2005) ressalta que o 
desenvolvimento de uma ciência se divide em fases, que vão desde a sua emergência, que corresponderia a $41 \%$ de sua vida, $33 \%$ ao seu crescimento uniforme e $26 \%$ ao seu desdobramento em ramos uniformes. O surgimento de novas disciplinas encontra na interdisciplinaridade os fundamentos necessários e é nela que se afirma, como reflexão epistemológica, a divisão do saber em disciplinas para extrair suas relações de interdependência e de conexões recíprocas (JAPIASSU, 1976 apud PINHEIRO, 2005).

Porém, talvez seja a ambiguidade do termo informação, quer dizer a diversidade de conceitos que determinam este termo, que façam com que a epistemologia da Clnf tenha também entendimentos diversos. Talvez seja a sua trajetória, suas origens ou ainda os paradigmas (conforme o filósofo Thomas Kuhn) dos diversos pesquisadores ou grupo destes, que acabem mostrando as diversas facetas das quais possam se olhar e analisar a Clnf. Adicione-se ainda o rápido desenvolvimento tecnológico das comunicações e dos meios informacionais que provocaram os mais diversos fenômenos, evoluções e até revoluções da forma e do entendimento das diversas dimensões da informação e, por conseguinte, a ciência que a estuda.

\section{ORIGENS DA CIÊNCIA DA INFORMAÇÃO (CINF)}

A análise das origens de uma ciência pode mostrar seus principais elementos. $O$ vetor formado pela trajetória no tempo pode apontar para onde ela sempre quis estar ou ser (desenvolver). Não é diferente com a CInf, ela não nasceu pronta e nem abruptamente. Segundo Shera e Cleveland (1977), o caminho histórico que fez surgir a CInf como ela é hoje, constitui-se de um emaranhado de fios. Um destes fios teve seu início nos primeiros bibliógrafos, em Alexandria, na biblioteca fundada por Ptolomeu, e caminhou para uma estruturação da bibliografia sistemática. Posteriormente, Paul Otlet e Henri La Fontaine realizaram um encontro histórico em Bruxelas em 1892, formaram o Instituto Internacional de Bibliografia (IIB) e apresentaram seus planos para uma compilação internacional de informação bibliográfica registrada, proporcionando um marco teórico para o que viria ser chamado de Documentação. Em 1950, no Instituto de Organização Bibliográfica, foi 
apresentado resultados de um estudo das tentativas de organizar a bibliografia internacional. A magnitude dessas iniciativas, em especial as do grupo de Bruxelas e de seus sucessores, exigiu um órgão totalmente novo de técnicas de organização, análise de assunto, descrição bibliográfica, e anotação que marcadamente diferem da prática biblioteca convencional. Justamente esta nova tecnologia veio a ser chamado de documentação e compõe uma importante vertente da CInf.

Já Pinheiro (2005) apresenta que a CInf é decorrente de uma sequência de eventos científicos e que conforme Harmon (1971 apud PINHEIRO, 2005) foi estabelecida em fases: emergência, crescimento uniforme e desdobramento em ramos uniformes. Segundo Pinheiro (2005), diferentes autores apontam como nascimento da Clnf as reuniões do Georgia Institute for Technology e, neste cenário, ganha força o Relatório Weinberg (1963), que proclamava a transferência da informação como parte inseparável da pesquisa e desenvolvimento.

Ainda segundo Pinheiro (2005), a Clnf nasce, evolui e se consolida como fruto de circunstâncias históricas e sociais que anunciam o seu surgimento (pós II Guerra) juntamente com reuniões científicas - Royal Society (1948), Conferência de Informação Científica, em Washington (1958) e Georgia Institute for Technology (1961).

Enquanto isso, no Brasil, Pinheiro (2005) destaca que os estudos bibliográficos se iniciaram com o diretor da Biblioteca Nacional de 1870 a 1882, Benjamin Franklin Ramiz Galvão. Posteriormente o Instituto Brasileiro de Bibliografia e Documentação (IBBD), foi criado em 1954, como órgão do então Conselho Nacional de Pesquisas (CNPq). A criação deste Instituto constituía uma ação de vanguarda, dado que apenas dois anos antes, a Rússia, que era à época um dos países mais desenvolvidos, criou o VINIT, órgão similar ao IBBD. A criação do IBBD se deu por influência externa, por meio da UNESCO, em ação conjunta com a Fundação Getúlio Vargas (FGV), que estimulou a implantação do Instituto. Desde a sua criação como IBBD, até o ano de 1976, já denominado IBICT, o Instituto tinha os seguintes objetivos: (1) promover a criação e o desenvolvimento dos serviços especializados de bibliografia e 
documentação; (2) estimular o intercâmbio entre bibliotecas e centros de documentação, no âmbito nacional e internacional; (3) incentivar e coordenar o melhor aproveitamento dos recursos bibliográficos e documentários do país, tendo em vista, em particular, sua utilização pela comunidade científica e tecnológica. No Brasil, a década de 70 marca a institucionalização da área no País, tanto pela criação do primeiro mestrado em Ciência da Informação, no IBICT, em 1970, com mandato acadêmico da UFRJ, quanto de outros cursos de pós-graduação que se seguiram, embora o do IBICT tenha sido o único, até a década de 90, em Ciência da Informação, área para a qual se voltaram os demais, nos anos 90. Segundo Pinheiro (2005), de modo geral, no exterior predominam as disciplinas de cunho tecnológico (sistemas de informação, tecnologias de informação e sistemas de recuperação da informação), enquanto no Brasil, os temas de maior interesse não têm esse caráter (Teoria da Ciência da Informação; Bibliometria; Representação da informação; Política de informação e Necessidade e usos de informação).

\section{ABRANGÊNCIA DA CIÊNCIA DA INFORMAÇÃO}

O conceito do Instituto de Tecnologia da Geórgia (1962/63) apresentado por Shera e Cleveland (1977) e Borko (1968, p.3) mostra que a Ciência da Informação é uma disciplina que investiga as propriedades e o comportamento informacional, as forças que governam os fluxos de informação, e os significados do processamento da informação, visando à acessibilidade e a usabilidade ótima. A Ciência da Informação está preocupada com o corpo de conhecimentos relacionados à origem, coleção, organização, armazenamento, recuperação, interpretação, transmissão, transformação, e utilização da informação. Isto inclui a pesquisa sobre a representação da informação em ambos os sistemas, tanto naturais quanto artificiais, o uso de códigos para a transmissão eficiente da mensagem, bem como o estudo do processamento e de técnicas aplicadas aos computadores e seus sistemas de programação. É uma ciência interdisciplinar derivada de campos relacionados, tais como a Matemática, Lógica, Linguística, Psicologia, Ciência da Computação, Engenharia da Produção, Artes Gráficas, Comunicação, Biblioteconomia, 
Administração, e outros campos científicos semelhantes.

Japiassu (1976 apud PINHEIRO, 1998) comenta que a Interdisciplinaridade, citada como característica no conceito do Instituto de Tecnologia da Geórgia é inerente às Ciências humanas e surge da necessidade de criar um fundamento a novas disciplinas. A interdisciplinaridade se afirma como reflexão epistemológica sobre a divisão do saber em disciplinas para extrair suas relações de interdependência e de conexões recíprocas. Sua grande esperança é a renovação e a mudança no domínio da metodologia das ciências humanas seu objetivo ideal é descobrir as leis estruturais de sua constituição e funcionamento de seu denominador comum. Ainda segundo Pinheiro (1998) os estudos de interdisciplinaridade da CInf frequentemente abrangem teorias, e não propriamente áreas, especialmente a teoria da informação e a Teoria Geral de Sistemas (TGS). Outro ponto importante, na visão de Pinheiro (1998), é que a Ciência da Informação tem como nascente o processo de comunicação e informação que se desenvolve em diferentes territórios: científicos, tecnológicos, educacionais, sociais, artísticos e culturais, portanto, múltiplos contextos e condições experimentais.

Com relação a Documentação, Shera e Cleveland (1977) consideram como uma parte essencial de nosso mundo moderno, um sistema de comunicação gráfica no mundo acadêmico, e incluiu os instrumentais dispositivos previstos para acelerar o fluxo de informações dentro de um grupo de especialistas, ou entre diferentes grupos de especialistas. Neste ponto eles consideram que a Documentação não está preocupada com o fluxo de informações no nível popular. A tarefa essencial da Documentação pode ser descrita como a combinação de dois padrões; o padrão de todas as atividades acadêmicas nas quais o uso de registros gráficos primários desempenha uma parte e o padrão dos serviços de intermediação que transmitem primeiro material gravado do estudioso como produtor para o estudioso como usuário. Porém, organização bibliográfica está como causa da canalização de todos os registros gráficos, para todos os usuários, para todos os efeitos a todos os níveis, de forma a otimizar a utilização social desses materiais como os 
registros da experiência humana, contrapondo a visão de foco só em especialistas.

Com relação aos enfoques da CInf, correlacionados com os significados da palavra informação, Borges et al (2003) apresentam como um dos desafios da CInf apresentar respostas às questões acerca do que é conhecimento e o que é informação. O conceito e a abrangência da Ciência da Informação têm se modificado ao longo do tempo. Alguns destes marcos foram a Teoria Matemática da Comunicação, em 1948, por Claude Shannon e a clássica definição de Ciência da Informação dada por Borko (1968). A conferência do Georgia Institute of Technology (1962), Relatório Weinberg (1963), Trabalho de Mikhailov sobre informática (1966). A definição sintetizada por Borko (1968, p. 3) segundo Pinheiro (1998) é uma das primeiras definições de Taylor (1966), que compôs o grupo que formulou a definição pelo Instituto de Tecnologia da Georgia, apresentada em definitivo artigo em torno do que seria CInf (já citado anteriormente neste trabalho na introdução e no início desta seção).

No entanto, segundo Borges et al (2004), a situação alterou-se por volta dos anos 70 onde o enfoque da CInf passou a ser também o usuário. A 'nova' Ciência da Informação, constituída, agora, como uma ciência social, focaliza-se nos usos e necessidades de informação. Solicita a contribuição das ciências cognitivas, não apenas os aspectos do comportamento do usuário, mas, também, aspectos relacionados com os estudos sobre o cérebro e a mente. $\mathrm{Na}$ sua interdisciplinaridade a CInf passa contar com a Ciência Cognitiva além Biblioteconomia, Comunicação, Ciência da Computação (SARACEVIC, 1996). Nesse modelo a informação precisa ser captada (no sentido de coletada), representada por meio de símbolos, ficar armazenada nas memórias primária ou secundária, para posterior processamento (manipulação de símbolos) que, eventualmente, poderia alterar o estado de conhecimento do sujeito. A noção de representação de coisas - objetos ou eventos - do mundo real no mundo da mente ocupa um lugar central na ciência da cognição tradicional. De fato, a informação é o ponto de apoio essencial de toda a ciência cognitiva tradicional e de todas as demais disciplinas do conhecimento que, de alguma forma, tem a informação e o conhecimento entre os seus objetos de estudo. E a partir disto 
as ideias que entranham na Ciência da Informação: construção de modelos mentais de usuários de informação, Mapas cognitivos de tomadores de decisão, Modelagem do processo de decisão, etc. (MOSTAFA; MOREIRA, 2012). Pinheiro (1998) corrobora este aspecto de inclusão da Ciência Cognitiva e completa dizendo que a Ciência da Informação tem seu próprio estatuto científico, como ciência social que é, portanto, interdisciplinar por natureza, e apresenta interfaces com a Biblioteconomia, Ciência da Computação, Ciência Cognitiva, Sociologia da Ciência e Comunicação, entre outras áreas, e suas raízes, em princípio, vêm da bifurcação da Documentação/Bibliografia e da Recuperação da Informação.

\section{INTELIGÊNCIA DE NEGÓCIOS (IN) E INTELIGÊNCIA COMPETITIVA (IC)}

Inteligência de Negócios (IN) mais conhecida pelo seu termo em Inglês Business Intelligence (BI) pode ser conceituado como um termo contemporâneo usado para se referir a um conjunto de tecnologias de informação (plataformas, aplicações e processos), que visa facilitar a tomada de decisões em todos os níveis (TODESCO et al, 2007). Na Clnf existe uma ambiguidade terminológica principalmente com o conceito de Inteligência Competitiva. A simplificação proposta na Introdução permite tirar vantagem disto e utilizar as análises epistemológicas da Inteligência Competitiva, mais estudada pela CInf, e utilizá-las como validas também para IN.

Existem alguns artigos científicos sobre a Epistemologia da $\mathrm{Cl}$ e a Inteligência Competitiva. Um deles, o de Capuano et al (2009), apresenta o artigo de Menezes (2005) que consiste em uma revisão de literatura sobre Inteligência Competitiva em periódicos da Clnf e mostra uma convergência para dois aspectos que estabelecem a conexão com a CInf. Os dois aspectos são: (1) o processo de tomada de decisão nas organizações e (2) a interação informacional da organização com o ambiente externo. Capuano et al (2009) continuam sua análise destacando que o primeiro ponto, apesar de estudado há muito pela administração, como nas obras seminais de Simon (1976) e Argyris (1978 e 1991), tem despertado atenções e motivado linhas de pesquisa na CInf inspiradas em trabalhos de autores mundialmente reconhecidos, tais 
como Choo (2003; 1998; 1991), Nonaka e Takeuchi (1997) e Daft e Weick (1984). E, que o segundo ponto, tem sido tratado principalmente nas publicações de Davenport (2000), McGee e Prusak (1994), Porter (1986) e outros.

Estes aspectos convergentes ou comuns, de certa forma demonstram a inter-relação entre os conceitos de Inteligência nas organizações. Capuano et al (2009) discute a integração de vários conceitos de inteligência corporativa e traz a obra de Liebowitz (2006) como não sendo a única a tratar esta integração. Nesta obra, Liebowitz (2006 apud CAPUANO et al, 2009) apresenta a ideia de consolidação dos conceitos de business intelligence (BI), inteligência competitiva e gestão do conhecimento (GC) no mesmo framework de gestão da informação e do conhecimento e que o autor denomina Inteligência Estratégica (IE).

Capuano et al (2009) também apresentam uma síntese epistemológica contemporânea útil acerca dos processos de tomada de decisão nas organizações, encontrada Choo (2003):

O conhecimento organizacional é uma propriedade coletiva da rede de processos de uso da informação, por meio dos quais os membros da organização criam significados comuns, descobrem novos conhecimentos e se comprometem com certos cursos de ação. O conhecimento organizacional emerge quando os três processos de uso da informação - criação de significado, construção do conhecimento e tomada de decisões - se integram num ciclo contínuo de interpretação, aprendizado e ação. (CHOO, 2003, p. 420, grifo nosso).

A interdisciplinaridade no conceito de Japiassu (1976, p. 5) também traz elementos para a integração dos conceitos e sua pertinência com os dois aspectos de conexão com a CInf. Silva, Dib e Silva (2011) apresentam o elemento informação, na sua condição de artefato e substrato de todas as ciências, como elemento primordial da característica interdisciplinar da Clnf e condição epistemológica essencial à integração com outras disciplinas, como a IC e a GC. Também apresenta Pinheiro (2006) que mapeou o campo interdisciplinar da área e constatou que, desde a década de 90, a 
Administração mantém uma estreita relação interdisciplinar com a Clnf, pela utilização de conceitos comuns, como Sistemas de Informação, Políticas de Informação, Gestão da Informação, Gestão do Conhecimento e Inteligência Competitiva.

Como expressado anteriormente, a simplificação dos elementos essenciais contidos na IN e também na visão integrada dos diversos conceitos de inteligência em organizações permite que os elos sejam explicitados para que através então de uma análise epistemológica da própria CInf encontre seu encaixe e sua inserção neste campo científico.

\section{POTENCIALIDADES DA CIÊNCIA DA INFORMAÇÃO}

Da mesma forma que no aspecto da abrangência, a dimensão da interdisciplinaridade se mostra importante também na potencialidade e o faz conforme mostra Pinheiro (1998). É pois, do conjunto de disciplinas que compõem a Ciência da Informação que pode ser pensada a interdisciplinaridade, isto é, de que forma e como outros campos do conhecimento contribuem para a Ciência da Informação, com seus conceitos, princípios, técnicas, métodos e teorias e, inversamente, a Ciência da Informação para os demais campos do conhecimento.

Segundo Borges et al (2004) é incontestável que, como campo epistemológico, a Ciência da Informação tem evoluído no que diz respeito às tendências e enfoques de pesquisa. A Ciência da Informação tem experimentado algumas mudanças paradigmáticas. As abordagens contemporâneas sobre a cognição fornecem possibilidades inovadoras para a discussão sobre a informação e o conhecimento, no âmbito da Ciência da Informação, por romperem com o paradigma da objetividade ao considerarem o sujeito no meio, mas também com as muitas e, frequentemente, falsas dicotomias - sujeito/objeto, sujeito/ação, parte/todo, razão/emoção - até então fortemente presentes nas vertentes tradicionais das ciências cognitivas. Basicamente, as concepções cognitivas contemporâneas consideram que somos seres individuais e seres sociais ao mesmo tempo, a todo instante, de forma absolutamente indissociável, o que modifica sobremaneira os conceitos 
que prevalecem a respeito da informação e do conhecimento na Ciência da Informação (BORGES et al, 2004).

Em seu artigo sobre 'O que é Ciência da Informação?', Araújo (2014) apresenta a visão de paradigmas da Clnf, de acordo com Capurro (2003). Segundo Capurro (2003) o paradigma físico foi a base para o nascimento da CInf. Este paradigma se estabelece a partir da Teoria Matemática de Shannon e Weaver. Nele, no paradigma, está contida a visão de que a informação é 'algo', um objeto físico, que um emissor transmite a um receptor. O segundo paradigma, o cognitivo, nasceu nos anos 1970, inspirado na teoria dos 'três mundos' de Karl Popper. Tal modelo relaciona informação a conhecimento: algo é informacional na medida em que altera as estruturas de conhecimento do sujeito que se relaciona com dados ou documentos. Mais recentemente, estaria emergindo um paradigma social, voltado para a constituição social dos processos informacionais. A partir da crítica ao modelo anterior, que via o usuário como um ser isolado da realidade e apenas numa dimensão cognitiva, busca-se aqui reinseri-lo nos seus contextos concretos de vida e atuação, numa perspectiva claramente fenomenológica: ver os sujeitos como 'ser no mundo', tal como a fórmula da existência (dasein) tomada de Heidegger ou as 'comunidades de discurso' estudadas por Hjorland e Albrechtsen a partir de uma inspiração em Wittgenstein. Daí a famosa fórmula de Capurro, onde a informação não é a matéria-prima do conhecimento: antes, é apenas a existência de um conhecimento partilhado entre diferentes atores que faz com que algo seja reconhecido como 'informação' (ARAÚJO, 2014).

Com base nestes paradigmas, Araújo (2014) descreve três modelos de estudo da Informação: (a) informação como fenômeno objetivo, (b) Abordagem subjetiva da informação e o (c) Ato de IN-FORMAR. No modelo (a) o foco da CInf está voltado para a construção de modelos e sistemas que garantam um transporte mais rápido, mais barato e mais eficiente das mensagens ou sinais que são trocados entre diferentes sujeitos e, mesmo tendo sido o primeiro modelo adotado pela área, mantém sua atualidade, sobretudo na conformação dos motores de busca na internet. Já no modelo (b), com a conformação do modelo 'cognitivo' a partir dos finais dos anos 1970, a Clnf não se tornou uma 
'ciência dos conhecimentos', uma 'ciência das ideias', isto é, uma ciência daquilo que estaria 'dentro da mente' das pessoas. Pelo contrário, a área manteve também um olhar próprio sobre esses fenômenos, buscando uma dimensão informacional no processo. Por último, o modelo (c) mais atual, no âmbito da perspectiva contemporânea pragmatista, inserem-se o contexto sociocultural e a dimensão interacional dos sujeitos no escopo do objeto de estudo do campo. Mas, a Clnf não é a ciência dos contextos socioculturais e nem a ciência das interações entre os sujeitos. Ela busca, nesses elementos, algo de específico, de particular: a dimensão informacional presente nesses fenômenos. É, mais uma vez, aí que reside o olhar informacional, o lugar de onde a Clnf observa os diferentes fenômenos da realidade. Neste modelo (c) agrupando as classificações dos diferentes autores citados, pode-se dizer que emerge um conceito INTERSUBJETIVO de informação (ARAÚJO, 2014).

\section{CONSIDERAÇÕES FINAIS}

Mesmo com a diversidade conceitual de Inteligência de Negócios (IN), o consenso sobre os seus elementos fundamentais: informação relevante ou estratégica, apoio a tomada de decisão e a gestão de informações de suporte permitem uma abordagem pela Clnf. A interdisciplinaridade e o corpo conceitual da definição de Clnf apresentado pelo Instituto de Tecnologia da Geórgia (1962/63) tem amplitude suficiente para amparar cientificamente pesquisa em IN em boa parte dos seus aspectos. Isto significa que através da interdisciplinaridade, as teorias, os pesquisadores e aplicações, conseguem promover a acessibilidade e usabilidade ótimas em IN. Os três paradigmas da CInf, físico, cognitivo e pragmático, segundo Capurro (2003 apud o ARAÚJO, 2014), também envolvem a informação de IN e desta forma permitem, conforme Araújo (2014), dos modelos de estudo: (a) informação como fenômeno objetivo, (b) Abordagem subjetiva da informação e o (c) Ato de INFORMAR.

Finalmente é possível relacionar ainda mais especificamente IN com a CInf em seu paradigma Cognitivo. Como apresentado por Saracevic (1996) a CInf na sua interdisciplinaridade passa contar com a Ciência Cognitiva. E, 
segundo Borges et al (2004), por volta dos anos 70 o enfoque da Clnf passou a ser também o usuário. A Clnf focaliza-se nos usos e necessidades de informação. Solicita a contribuição das ciências cognitivas, não apenas os aspectos do comportamento do usuário, mas também aspectos relacionados com os estudos sobre o cérebro e a mente. A informação não mais estudada somente pelo seu aspecto físico, mas mudando o estado de conhecimento do usuário e apoiando a tomada de decisões. Araújo (2014) confirma então que o paradigma cognitivo, inspirado na teoria dos 'três mundos' de Karl Popper, relaciona informação a conhecimento: algo é informacional na medida em que altera as estruturas de conhecimento do sujeito que se relaciona com dados ou documentos.

\section{REFERÊNCIAS}

ARAÚJO, C. A. Á. O Que é ciência da informação? Informação \& Informação, Londrina, v. 19, n. 1, p. 01-30, jan./abr. 2014. Disponível em: $<$ http://www.uel.br/revistas/uel/index.php/informacao/article/view/15958>. Acesso em: 20 mar. 2015.

BORGES, M. E. N. et al. A ciência da informação discutida à luz das teorias cognitivas: estudos atuais e perspectivas para a área. Cadernos Bad, n.2, 2004.

BORKO, H. Information Science: What is it? American Documentation, v. 19, n. 1, p.3-5, jan. 1968.

CAPUANO, E. A. et al. Inteligência competitiva e suas conexões epistemológicas com gestão da informação e do conhecimento. Ciência da Informação, Brasília, v. 38, n. 2, p. 19-34, 2009.

CAPURRO, R. Epistemologia e Ciência da Informação. IN: ENCONTRO NACIONAL DE PESQUISA EM CIÊNCIA DA INFORMAÇÃO, 5., 2003. Belo Horizonte. Anais Eletrônicos... Belo Horizonte: ENANCIB, 2003.

CHOO, C. W. A organização do conhecimento. São Paulo: SENAC, 2003.

GARTNER. Disponível em: <https://www.gartner.com/technology/about.jsp>. Acesso em: 20 mar. 2015.

GRECO, J. O que é epistemologia. Compêndio de Epistemologia. São Paulo: Loyola, 2008.

JAPIASSU, H. Interdisciplinaridade e patologia do saber. [S.I]: Imago, 1976. 
MENEZES, E. M. Inteligência competitiva: uma revisão de literatura competitive intelligence: literature review. Revista Digital de Biblioteconomia \& Ciência da Informação, v. 3, n. 1, p. 103-130, 2005.

MOSTAFA, S. P.; MOREIRA, W. Referenciais teóricos da área de informação: sobre Isa e Vânia para os professores da ABEBD. Transinformação, v. 11, n.1, 2012.

PINHEIRO, L. V. R. Evolução e tendências da Ciência da Informação, no exterior e no Brasil: quadro comparativo a partir de pesquisas históricas e empíricas. Enancib, v. 2004, p. 1-12, 2005. Disponível em:

$<$ http://www.ancib.org.br/media/dissertacao/GT1 Pinheiro.pdf $>$. Acesso em: 20 mar. 2015.

Campo interdisciplinar da Ciência da Informação: fronteiras remotas e recentes. Investigación Bibliotecológica, México, v.12, n.25, p. 132-163, 1998.

SANTOS, M. Y.; RAMOS, I. Business intelligence: tecnologias da informação na gestão do conhecimento. Lisboa: FCA, 2006.

SARACEVIC, T. Ciência da informação: origem, evolução e relações.

Perspectivas em Ciência da Informação, v. 1, n. 1, p. 41-62, 1996.

Disponível em:

$<$ http://portaldeperiodicos.eci.ufmg.br/index.php/pci/article/view/235>. Acesso em: 21 mar. 2015.

SHERA, J. H.; CLEVELAND, D. B. History and foundations of Information Science. Annual Review of Information Science and Technology (ARIST). Washington. n. 12, p. 249-275, 1977.

SHOLLO, A.; GALLIERS, R. D. Towards an understanding of the role of business intelligence systems in organisational knowing. Information Systems Journal, v. 26, n. 4. Jul. 2015.

SILVA, S. A.; DIB, S. F.; SILVA, N. C. Ciência da informação: relação interdisciplinar com as disciplinas inteligência competitiva e gestão do conhecimento. In: ENCONTRO NACIONAL DE PESQUISA EM CIÊNCIA DA INFORMAÇÃO, 12., 2011, Brasília, Anais... Brasília: UnB, 2011.

TODESCO, J. L.; CARRETERO, L. E.; DURAN, A. Business Intelligence (Business Intelligence). [Slides]. In: CURSO DE BUSINESS INTELLIGENCE. Escuela Complutense Latinoamericana, Florianópolis, 2007.

TURBAN, E.; RAMESH, E. S.; DELEN, D. Decision support and business intelligence systems. 9 ed. Upper Saddle River, NJ, USA: Prentice Hall Press, 2010. 


\title{
BUSINESS INTELLIGENCE AND ITS EPISTEMOLOGICAL CONDITION IN INFORMATION SCIENCE
}

\begin{abstract}
Introduction: Business Intelligence $(\mathrm{BI})$ is a term frequently used in quite different worlds and, thus, inaccurate in relation to its definition and its epistemological boundaries. Information science traditionally investigated the information behavior and the information properties, the forces that control the flow of information, and the meanings of information processing aiming at accessibility and optimal usability. In this sense, one wonders if Information Science is supported epistemologically to contribute to the development of the Business Intelligence approaches.

Objective: The objective of this study is to identify the epistemological foundations that allow dealing with Business Intelligence in the field of Information Science.

Methodology: The exploratory study seeks to extend the understanding of the topic through a conceptual review of origins, epistemology and scope of Information Science and the interconnections with Business Intelligence.

Results: The result identifies the potential of Information Science regarding Business Intelligence through its paradigms and knowledge study models. Conclusions: The cognitive paradigm - its study model, researchers and theories of information - is presented as the most specifically suitable for the epistemological support of Business Intelligence in the field of Information Science.
\end{abstract}

Descriptors: Business Intelligence. Information Science. Epistemology.

\section{BUSINESS INTELLIGENCE Y SU CONDICIÓN EPISTEMOLÓGICA EN CIENCIAS DE LA INFORMACIÓN}

\section{RESUMEN}

Introducción: La Inteligencia de Negocios o Business Intelligence (BI), es un término ampliamente utilizado en diferentes campos y presenta imprecisiones sobre su definición y sus límites epistemológicos. La Ciencia de la Información ha tradicionalmente investigado las propiedades y el comportamiento informativo, las fuerzas que gobiernan los flujos de información y los significados del procesamiento de la información con el objetivo de obtener una óptima usabilidad y accesibilidad. En este sentido, la pregunta es: ¿cuenta la ciencia de la información con soporte epistemológico suficiente como para contribuir al desarrollo de enfoques de Inteligencia de Negocios?

Objetivo: El objetivo de este estudio es identificar los fundamentos epistemológicos que permiten abordar la Inteligencia de Negocios en el campo de la Ciencia de la Información.

Metodología: El estudio, de naturaleza exploratoria, busca ampliar la comprensión del 
tema por medio de una revisión conceptual sobre los orígenes, la epistemología y la amplitud de la Ciencia de la Información y las interconexiones con la Inteligencia de Negocios.

Resultados: El resultado identifica el potencial de las Ciencia de la Información para con la Inteligencia de Negocios a través de sus paradigmas y modelos de estudio de los conocimientos.

Conclusiones: El paradigma cognitivo - su modelo de estudio, los investigadores y las teorías de la información - es presentado como lo más apropiado para el apoyo epistemológico en el campo de las Ciencias de la Información.

Descriptores: Business Intelligence. Ciencia de la información. Epistemología. 